JULIEN WAEYTENS AND BoJAna V. Rosić

\title{
COMPARISON OF \\ DETERMINISTIC AND \\ PROBABILISTIC APPROACHES \\ TO IDENTIFY THE DYNAMIC \\ MOVING LOAD AND DAMAGES \\ OF A REINFORCED CONCRETE \\ BEAM
}

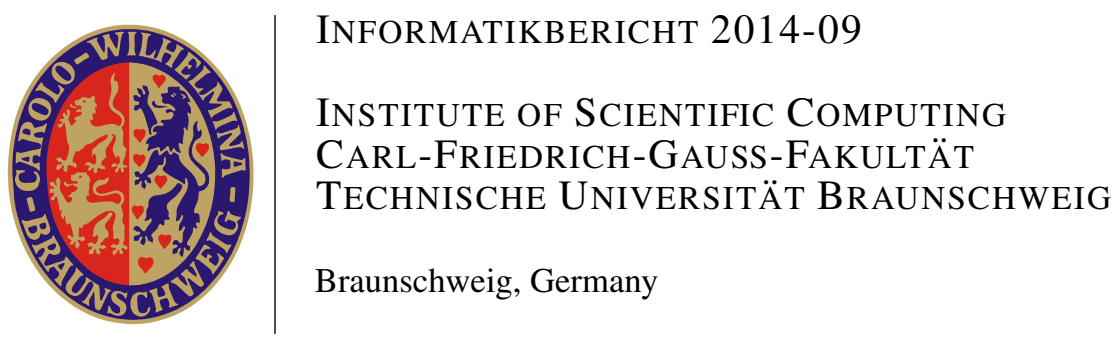


This document was created February 2014 using $\mathrm{LT}_{\mathrm{E}} \mathrm{X} 2 \varepsilon$.

Institute of Scientific Computing

Technische Universität Braunschweig

Hans-Sommer-Straße 65

D-38106 Braunschweig, Germany

e-mail: wire@tu-bs.de

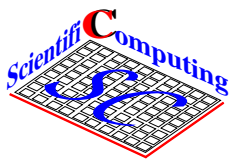

url: www.wire.tu-bs.de

Copyright (C) by Julien Waeytens and Bojana V. Rosić

This work is subject to copyright. All rights are reserved, whether the whole or part of the material is concerned, specifically the rights of translation, reprinting, reuse of illustrations, recitation, broadcasting, reproduction on microfilm or in any other way, and storage in data banks. Duplication of this publication or parts thereof is permitted in connection with reviews or scholarly analysis. Permission for use must always be obtained from the copyright holder.

Alle Rechte vorbehalten, auch das des auszugsweisen Nachdrucks, der auszugsweisen oder vollständigen Wiedergabe (Photographie, Mikroskopie), der Speicherung in Datenverarbeitungsanlagen und das der Übersetzung. 


\title{
Comparison of deterministic and probabilistic approaches to identify the dynamic moving load and damages of a reinforced concrete beam
}

\author{
Julien Waeytens ${ }^{1}$ and Bojana V. Rosić ${ }^{2}$ \\ ${ }^{1}$ IFSTTAR, Université Paris-Est \\ ${ }^{2}$ Institute of Scientific Computing, TU Braunschweig \\ julien.waeytens@ifsttar.fr \\ bojana.rosic@tu-bs.de
}

Two classical civil engineering inverse problems are considered. The first deals with the determination of dynamic moving loads applied to a reinforced concrete beam. The second one corresponds to the monitoring and the damage assessment. The concrete damage due to overloading is modelled by a loss of the concrete Young' modulus, whereas the steel bar damage due to corrosion effects is modelled by a reduction of the steel bar cross section. To identify the loading and damage parameters, deterministic and probabilistic model updating techniques are applied and compared. In the deterministic approach, a gradient descent technique based on adjoint framework is used to minimize the data misfit functional with a Tikhonov regularization term. Regularization by means of Bayes rule is considered in a probabilistic approach. The estimation is of the minimum variance type achieved with the help of the transformed ensemble Kalman filter. 
4

http://www.digibib.tu-bs.de/?docid=00057886 


\section{Contents}

1 Introduction $\quad 6$

2 Model problem $\quad 7$

2.1 Study 1: Identification of the moving load . . . . . . . . . . . 8

2.2 Study 2: Determination of the material parameters . . . . . . . 8

3 Identification procedures $\quad 9$

3.1 Deterministic approach . . . . . . . . . . . . . . . 10

3.2 Probabilistic approach . . . . . . . . . . . . . . 12

4 Numerical result comparison $\quad 14$

4.1 Study 1: Identification of the moving load . . . . . . . . . . . 15

4.1.1 Load identification using simple mechanical framework . . 15

4.1.2 Load identification in 2D elastodynamics . . . . . . . . . 17

4.2 Study 2: Determination of the material parameters . . . . . . . 18

5 Conclusions 23

$\begin{array}{lr}\text { References } & 24\end{array}$ 


\section{Introduction}

Structural Health Monitoring is particularly useful to detect and localize damages, to reduce the maintenance cost of structures and to ensure the user safety. To prevent early damages, smart systems have been developed to identify overloaded vehicles on civil engineering structures such as bridges and viaducts. Particular example of these is the Bridge Weigh-In-Motion (B-WIM) system that has been studied for the last 30 years $[16,17,8]$. Concerning the damage identification, according to [21] one may distinguish four categories: the detection of damage (level 1), localization (level 2), quantification of the damage (level 3) and lifetime prediction update (level 4). Categories of level 1 and 2 can be achieved with the help of the data driven methods, such as for example the vibration-based techniques. For this purpose one can use modal-based [4, 3] or static based statistical approaches [18, 22]. To accomplish higher level categories, one requires model updating techniques. The parameter (model) estimation from the noisy indirect sensor outputs is not an easy task. The problem being generally ill-posed, requires certain kind of regularisation [25]. In the deterministic sense, the regularisation is very often achieved by Tikhonov regularization [26]. However, other possible techniques also exist such as for example Error in the Constitutive Relation (ECR) regularization [10, 2, 1] previously studied by author. On the other hand, the ill-posed problem can be regularised in a probabilistic manner via Bayes rule by adding the prior expert knowledge on the parameter (model) set next to the observation data. Most of these approaches are based on the Monte Carlo kind of sampling procedures such as for instance Markov Chain Monte Carlo techniques $[9,13]$. In the recent years, another more simple kind of methods appeared. These often assume linearity of the observation operator and Gaussian noise- the linear Bayesian filters—such as the ensemble Kalman filter [6] and its generalisation in the form of polynomial chaos based linear filter [19, 20]. In case of nonlinearity these can be extended to more complex and accurate forms as presented in $[5,11]$.

The objective of this paper is to qualitatively and not quantitatively compare deterministic [28] and probabilistic [19] updating techniques. For this purpose the Tikhonov regularisation and ensemble Kalman filter procedures are applied on two important civil engineering applications: the identification of a moving load on a reinforced concrete beam and the detection of damages in the concrete and steel bar of the beam. The identification is performed by using the full temporal data read by strain sensors and structural dynamic model based on partial differential equation.

The article is organized as follows: Section 2 summarises the motivation behind of this work and focuses on the description of two inverse civil engineering problems 
of huge practical importance. Section 3 lays out the mathematical dimension of the numerical approaches used in this research, and Section 4 tuys up the numerical findings of both deterministic and probabilistic computational approaches.

\section{Model problem}

A simplified model of a 2D concrete beam with a single horizontal steel bar is considered (see Fig. 1) in the time interval $[0, T]$. The steel bar and the concrete are supposed perfectly adherent. Under plain strain assumption, the mathematical formulation of the beam consists of the dynamic equilibrium:

$$
\begin{aligned}
& \int_{\Omega} \rho_{c} \underline{\ddot{u}} \cdot \underline{u}^{*} d \Omega+\int_{\Gamma} \rho_{b} S_{b} \underline{\ddot{u}} \cdot \underline{u}^{*} d \Gamma+\int_{\Omega} \underline{\underline{\varepsilon}}(\underline{u}): \mathscr{K}_{c}: \underline{\underline{\varepsilon}}\left(\underline{u}^{*}\right) d \Omega+ \\
& \int_{\Gamma} E_{b} S_{b}(\underline{x}) \frac{\partial u_{x}}{\partial x} \frac{\partial u_{x}^{*}}{\partial x} d \Gamma-\int_{\partial \Omega_{f}} \underline{F}_{d}(\chi, t) \cdot \underline{u}^{*} \partial \Omega=0, \quad \forall \underline{u}^{*} \in \mathscr{U}_{0}
\end{aligned}
$$

with zero initial conditions and the kinematic conditions satisfying $\underline{u} \in \mathscr{U}_{0}=\left\{\underline{u}^{*} \in\right.$ $H_{1}(\Omega) \backslash \underline{u}^{*}=\underline{0}$ on $\partial \Omega_{i 1}, \underline{u}^{*} \cdot \underline{y}=0$ on $\left.\partial \Omega_{i 2}\right\}$. In Eq. (1) $\rho_{c}$ (resp. $\rho_{b}$ ) represents the

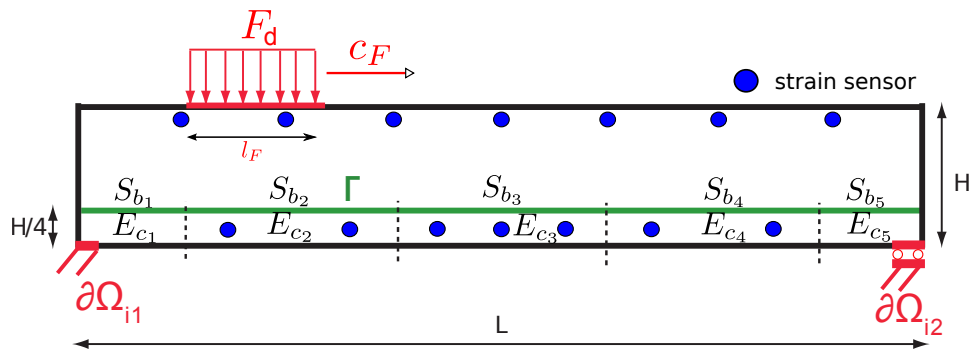

Figure 1: Concrete beam of dimensions $L=30 \mathrm{~m}$ and $H=1 \mathrm{~m}$ with a steel bar $\Gamma$ instrumented with strain sensors. $\underline{F}_{d}$ is dynamic moving load, $S_{b_{j}}$ $(j \in\{1, \ldots, 5\})$ is steel bar cross section assumed piecewise constant on each interval, $E_{c_{j}}(j \in\{1, \ldots, 5\})$ is concrete Young's modulus assumed piecewise constant on each subdomain.

volumic mass of the concrete (resp. steel bar), $S_{b}$ is the area of the steel bar crosssection, $E_{b}$ is the steel bar Young's modulus, and $\mathscr{K}_{c}$ is the concrete Hooke's tensor which depends on the Young's modulus $E_{c}$ and the Poisson ratio $v_{c}$. Lastly, $\underline{F}_{d}(\chi, t)$ represents the dynamic moving load applied to the top of the concrete beam. 
The beam is instrumented with fourteen strain sensors, seven on the upper and seven on the bottom part of the beam, which can monitor the traffic-induced strain responses of the beam. The strain is measured over a period of time $T=5 \mathrm{~s}$, and collected into the temporal data series, which further allow the determination of the dynamic moving loading, and hence detection of the overloaded vehicles causing the damage in the structure. To assess structural damages, the measurement data are also used for the identification of the material parameters such as Young's modulus and steel bar cross section.

\subsection{Study 1: Identification of the moving load}

To reduce the number of unknowns to be determined by the inverse modelling technique, the dynamic moving load is modelled as

$$
\underline{F}_{d}(\chi, t)=-F_{d} f\left(\chi, l_{F}\right) \underline{y} \quad, \quad \chi=x-c_{F} t,
$$

and hence parametrized by three scalars: the loading amplitude $F_{d}(\mathrm{~N} / \mathrm{m} 2)$, the loading velocity $c_{F}(\mathrm{~m} / \mathrm{s})$ and the loading length $l_{F}(\mathrm{~m})$, as shown in Fig. 1 . The function $f\left(\chi, l_{F}\right)$ represents the time dependent loading profile applied to the top of the beam, and here is assumed to follow a parabolic law.

For the further study the loading parameters $\left(F_{d}, c_{F}, l_{F}\right)$ are normalised to $\left(\bar{F}, \bar{c}_{F}, \bar{l}_{F}\right)$ with respect to their standard values $\left(F_{0}, c_{F}^{0}, l_{F}^{0}\right)$ such that

$$
F_{d}=\bar{F} F_{0} \quad, \quad c_{F}=\bar{c}_{F} c_{F}^{0} \quad, \quad l_{F}=\bar{l}_{F} l_{F}^{0}
$$

hold. In this manner, the normalized parameters are assumed to be unknown, whereas the standard loading parameters are used as an initial guess. The identification of $\left(\bar{F}, \bar{c}_{F}, \bar{l}_{F}\right)$ is performed by both techniques deterministic and probabilistic given the data read by seven equidistantly distributed strain sensors $S_{1}-S_{7}$, which are placed in the close vicinity to the top of the concrete beam. The sensors are distanced by $0.125 L$ and lie on the same vertical $7 H / 8$, in which $L$ and $H$ denote the length and height of the beam, respectively.

\subsection{Study 2: Determination of the material parameters}

According to the classical damage theory [14], the concrete damage is modelled by a loss of the concrete Young's modulus, and the corrosion effects are described by 
a reduction of the steel bar cross section. These material parameters are described by decomposing both the steel bar and the concrete zone into five sections. In each section $j$, the steel bar is represented by a constant value of cross-section $S_{b_{j}}$ and the concrete zone by a constant Young's modulus $E_{c_{j}}$. Note that a loss of Young's modulus in the concrete is only considered in a possible damaged area named $\Omega_{c}$, which corresponds to the bottom part of the 2D concrete beam. Similarly to before, the material parameters are normalised such that

$$
S_{b}(\underline{x})=\sum_{j=1}^{n_{b}} \bar{S}_{b_{j}} S_{b_{u d}} \phi_{j}^{s}(x) \quad, \quad E_{c}(\underline{x})=\sum_{j=1}^{n_{c}} \bar{E}_{c_{j}} E_{c_{u d}} \phi_{j}^{c}(x)
$$

hold. Here,

- $S_{b_{u d}}\left(\right.$ resp. $\left.E_{c_{u d}}\right)$ corresponds to the undamaged cross-section of the steel (resp. the undamaged Young modulus of the concrete);

- $\bar{S}_{b_{j}}$ (resp. $\bar{E}_{c_{j}}$ ) represents the $j^{\text {th }}$ normalized cross-section of the steel (resp. normalized Young modulus of the concrete) associated to the area $\Gamma_{j}$ (resp. $\Omega_{c_{j}}$ ) where $\phi_{j}^{b}(x)$ (resp. $\left.\phi_{j}^{c}(x)\right)$ is non-zero.

- $\phi_{j}^{b}(x)$ and $\phi_{j}^{c}(x)$ are basis functions on a coarse mesh grid $(\mathrm{H})$, chosen differently than the fine mesh grid (h) used in the finite element solver. The basis functions are a priori known. In practice, $\phi_{j}^{b}(x)$ (resp. $\left.\phi_{j}^{c}(x)\right)$ is a constant basis function equal to 1 in the area $\Gamma_{j}$ (resp. in the subdomain $\Omega_{c_{j}}$ ) and zero otherwise.

Furthermore, only the material parameters $\bar{S}_{b_{j}}$ and $\bar{E}_{c_{j}}$ are assumed to be unknown. They are estimated with the help of deterministic and probabilistic approaches by using the data read by seven equidistantly distributed strain sensors $S_{8}-S_{14}$ separated by the distance $L / 8$ and located in the bottom part of the concrete beam on the height $H / 8$.

\section{Identification procedures}

The problem of estimating either the moving loads or material characteristics corresponds to the reconstruction of a quantity from the noisy indirect observations. This mathematically coincides with the inversion of an operator, which is considered to be 
ill-posed problem in a sense of Hadamard. To resolve this issue many different procedures are advised in literature [27]. However, two of them have gained increased popularity in last few decades: the deterministic and probabilistic approaches. The deterministic regularisation procedures are trying to solve the issue of ill-posedness by approximating the inverse of the observation operator by a family of stable operators which depend on some regularisation parameter. An example is the Tikhonov regularisation procedure [26] further considered in this paper. On the other side, the probabilistic approach employs the Bayes rule [25] to assimilate the measurement data with the a priori chosen distribution-the regularisation parameter.

To show differences between these two phenomenological approaches, the problems described in the case study 1 and case study 2 are analysed and obtained results are compared. However, note that these methods cannot be really compared as they do not rely on the same assumptions and regularisation parameters. Therefore, the analysis further performed has for the main aim to illustrate and show applicability of both identification procedures.

\subsection{Deterministic approach}

By means of the optimal control theory, one aims at determining normalized scalars $\left(\bar{F}, \bar{c}_{F}, \bar{l}_{F},\left(\bar{E}_{c i}\right)_{i=1}^{n_{b}},\left(\bar{S}_{b j}\right)_{j=1}^{n_{c}}\right)$, further generally denoted by $q$, by minimizing the data misfit functional

$$
J(\underline{u} ; q)=\frac{1}{2} \sum_{i=1}^{n_{s}} \int_{0}^{T}\left[\int_{\Omega} \varepsilon_{x x}(u ; x, t) \psi_{i}\left(\underline{x}-\underline{x}_{i}\right) d \Omega-\left(\varepsilon_{x x}^{m e s}\right)_{i}(t)\right]^{2} d t+\|q\|
$$

in which the displacement field $\underline{u}$ satisfies the kinematic conditions defined in $\mathscr{U}_{0}$ and the dynamic equilibrium in Eq. (1). Note that the Tikhonov regularization term $\|q\|$ corresponds to

Study 1: $\|q\|=\varepsilon_{1}^{r e g} \frac{\alpha_{n_{3}}}{2}\left(\bar{F}^{2}+\bar{c}_{F}^{2}+\bar{l}_{F}^{2}\right)$,

Study 2: $\quad\|q\|=\varepsilon_{2}^{r e g} \frac{\alpha_{n_{1}}}{2} \int_{\Gamma}\left[\frac{S_{b}(x)}{S_{b_{u d}}}-1\right]^{2} d \Gamma+\varepsilon_{2}^{\text {reg }} \frac{\alpha_{n_{2}}}{2} \int_{\Omega}\left[\frac{E_{c}(x)}{E_{c_{u d}}}-1\right]^{2} d \Omega$.

Here, $\alpha_{n_{i}}, i \in\{1,2,3\}$ ensures the physical homogeneity of the terms in $J$ and $\varepsilon_{j}^{r e g}, j \in\{1,2\}$ is the regularization parameter determined using the discrepancy principle due to Morozov [15]. 
To solve the minimization problem at a low computation cost, the gradient method with the steepest descent direction obtained by the adjoint approach is utilised. This methodological procedure is taken due to equivalency of adjoint problems in case of loading and material parameter study. The process of solving the adjoint problem corresponds to a backward elastodynamics problem whose final conditions vanish. The goal is to search for the adjoint displacement $\underline{\tilde{u}} \in \mathscr{U}_{0}=\left\{\underline{u}^{*} \in H_{1}(\Omega) \backslash \underline{u}^{*}=\right.$ $\underline{0}$ on $\left.\partial \Omega_{i}\right\}$ such that:

$$
\begin{aligned}
& \int_{\Omega} \rho_{c} \ddot{\tilde{u}} \cdot \underline{u}^{*} d \Omega+\int_{\Gamma} \rho_{b} S_{b} \ddot{\tilde{u}} \cdot \underline{u}^{*} d \Gamma+\int_{\Omega} \underline{\underline{\varepsilon}}(\underline{\tilde{u}}): \mathscr{K}_{c} \underline{\underline{\varepsilon}}\left(\underline{u}^{*}\right) d \Omega \\
& +\int_{\Gamma} E_{b} S_{b} \frac{\partial \tilde{u}_{x}}{\partial x} \frac{\partial u_{x}^{*}}{\partial x} d \Gamma-\sum_{i=1}^{n_{s}} \int_{\Omega}\left(\tilde{\sigma}_{0_{x x}}\right)_{i} \frac{\partial u_{x}^{*}}{\partial x} d \Omega d t=0, \forall \underline{u}^{*} \in \mathscr{U}_{0}
\end{aligned}
$$

holds, where $\left(\tilde{\sigma}_{0_{x x}}\right)_{i}(\underline{x}, t)=\left[\left(\varepsilon_{x x}^{s i m}\right)_{i}(t)-\left(\varepsilon_{x x}^{m e s}\right)_{i}(t)\right] \psi_{i}\left(\underline{x}-\underline{x}_{i}\right), i \in\left\{1, . ., n_{s}\right\}$.

Once the direct and the adjoint states are evaluated, the gradient of the functional can be estimated at a low computation cost by means of the following formulas

$$
\begin{aligned}
\frac{\partial J}{\partial \bar{F}} & =-F_{0} \int_{0}^{T} \int_{\partial \Omega_{f}} f\left(\chi, l_{F}\right) \tilde{u}_{y} \partial \Omega d t \\
\frac{\partial J}{\partial \bar{c}_{F}} & =-F \int_{0}^{T} \int_{\partial \Omega_{f}} \frac{\partial f\left(\chi, l_{F}\right)}{\partial \bar{c}_{F}} \tilde{u}_{y} \partial \Omega d t, \\
\frac{\partial J}{\partial \bar{l}_{F}} & =-F \int_{0}^{T} \int_{\partial \Omega_{f}} \frac{\partial f\left(\chi, l_{F}\right)}{\partial \bar{l}_{F}} \tilde{u}_{y} \partial \Omega d t .
\end{aligned}
$$

for the identification of loads, and

$$
\begin{aligned}
\frac{\partial J}{\partial \bar{S}_{b j}}= & -\int_{0}^{T} \int_{\Gamma} E_{b} S_{b_{u d}} \phi_{j}^{b}(x) \frac{\partial u_{x}}{\partial x} \frac{\partial \tilde{u}_{x}}{\partial x} d \Gamma d t-\int_{0}^{T} \int_{\Gamma} \rho_{b} S_{b_{u d}} \phi_{j}^{b}(x) \underline{\ddot{u}} \cdot \underline{\tilde{u}} d \Gamma d t \\
& +\varepsilon_{1}^{r e g} \alpha_{n_{1}} \int_{\Gamma}\left(\frac{S_{b}(x)}{S_{b_{u d}}}-1\right) \phi_{j}^{b}(x) d \Gamma \\
\frac{\partial J}{\partial \bar{E}_{c j}}= & -\int_{0}^{T} \int_{\Omega} d \lambda_{D P} \phi_{j}^{c}(x) \operatorname{div}(\underline{u}) \operatorname{div}(\underline{\tilde{u}}) d \Omega d t \\
& -\int_{0}^{T} \int_{\Omega} 2 d \mu_{D P} \phi_{j}^{c}(x) \underline{\underline{\varepsilon}}(\underline{u}): \underline{\underline{\varepsilon}}(\underline{\tilde{u}}) d \Omega d t \\
& +\varepsilon_{1}^{r e g} \alpha_{n_{2}} \int_{\Omega}\left(\frac{E_{c}(x)}{E_{c_{u d}}}-1\right) \phi_{j}^{c}(x) d \Omega
\end{aligned}
$$

for the identification of material parameters. Here,

$$
d \lambda_{D P}=\frac{E_{c_{u d}} v_{c}}{\left(1+v_{c}\right)\left(1-v_{c}\right)}, d \mu_{D P}=\frac{E_{c_{u d}}}{2\left(1+v_{c}\right)}
$$


are Lame constants.

To summarise the deterministic model updating strategy, at each iteration the direct and adjoint problems are solved by taking the control parameters from the previous iteration. Given the direct and adjoint states, the functional gradient is computed. Lastly, the control parameters are updated.

\subsection{Probabilistic approach}

The deterministic strategy described in the previous section offers the best-guess estimates for the set of parameters $q$ without taking into account their uncertainty. To mitigate this, the probabilistic methods consider the unknown parameters as uncertain and provide solutions in terms of probability distributions. The distributions involve more information than deterministic estimates as they provide confidence intervals for the quantity being considered.

The level of parametric uncertainty depends upon their nature as well as expert knowledge gained through the subsequent practice and experience. For example, the Young's modulus $E_{c}$ is known to be positive definite quantity. According to the maximum entropy law, this makes the lognormal distribution the most suitable candidate for the description of this material constant.

By gathering all available prior information via elicitation techniques, the a priori probabilistic description of unknown quantities $q$ can be achieved. Mathematically, this corresponds to the modelling of $q$ with the help of the finite variance $\mathscr{Q}$-valued random variables (random fields) living in a probability space denoted by triplet $(\Omega, \mathbb{P}, \mathscr{B})$, in which $\Omega$ stands for the set of all events $\omega, \mathbb{P}$ is the probability measure and $\mathscr{B}$ is the Borel $\sigma$ algebra. In this space the random variable $q(\omega)$, or a prior, is defined as a mapping $q(\omega): \Omega \rightarrow \mathscr{Q}$ and associated with the expectation operator representing the high dimensional integral:

$$
\mathbb{E}(q)=\int_{\Omega} q(\omega) \mathbb{P}(d \omega) .
$$

By collecting the measurement data $\varepsilon_{x x}^{m e s}$, the prior knowledge about $q$ can be further updated (sharpened) with the help of Bayes rule:

$$
p\left(q \mid \varepsilon_{x x}^{m e s}\right)=p(q) \frac{p\left(\varepsilon_{x x}^{m e s} \mid q\right)}{p\left(\varepsilon_{x x}^{m e s}\right)},
$$


in which the description of parameter $q$ is conditioned on $\varepsilon_{x x}^{\text {mes }}$. Here, $p\left(q \mid \varepsilon_{x x}^{\text {mes }}\right)$ denotes the posterior conditional density of $q$ over $\varepsilon_{x x}^{\text {mes }}, p\left(\varepsilon_{x x}^{m e s} \mid q\right)$ describes how likely are the data $\varepsilon_{x x}^{m e s}$ given the prior $q$, and $p(q)$ is the prior density. Note that the data are related to the true value of parameter $q_{t}$ by

$$
\varepsilon_{x x}^{m e s}=Y\left(q_{t}\right)+\varepsilon
$$

in which $Y$ is a nonlinear operator, and $\varepsilon$ is the additive sum of measurement and model errors. This further means that the posterior density is conditioned by the measurement operator $Y$, i.e.

$$
p\left(q \mid \varepsilon_{x x}^{\text {mes }}\right)=p(q \mid Y(q)) .
$$

By not restricting oneself on only one piece of measurement, but defining Eq. (15) for all sub- $\sigma$-algebras, the previous relation can be transformed to the Kolmogorov conditional expectation equivalent to the orthogonal projection onto the space $\mathscr{Q}_{n}:=$ $\mathscr{Q} \otimes L_{2}(\Omega, \mathbb{P}, \sigma(Y))$, as shown in [19]. The last one further leads to the quadratic minimisation problem

$$
q_{a}(\omega):=\underset{\eta \in \mathscr{Q}_{n}}{\arg \min }\|q-\eta\|_{L_{2}}^{2}
$$

in which the unique solution can be expressed as $q_{a}(\omega)=\psi(Y)$, where $\psi \in$ $L_{0}(\mathscr{Y}, \mathscr{Q})$. By focusing only on the subset of linear continuous maps $\mathscr{L}(\mathscr{Y}, \mathscr{Q}) \subset$ $L_{0}(\mathscr{Y}, \mathscr{Q})$, the minimisation in Eq. (16) results in a generalisation of Gauss-Markov theorem [12]:

$$
q_{a}(\omega)=q_{f}(\omega)+K\left(\varepsilon_{x x}^{\text {mes }}(\omega)-\varepsilon_{x x}^{\text {sim }}(\omega)\right),
$$

in which $\varepsilon_{x x}^{s i m}(\omega)$ is the forecasted measurement, $\varepsilon_{x x}^{\text {mes }}(\omega)$ is the sensor output and $K$ is the Kalman gain. The gain is obtained as product of corresponding covariances functions (here denoted by $C$ )

$$
K=C_{q_{f}, \varepsilon_{x x}^{s i m}}\left(C_{\varepsilon_{x x}^{s i m}, \varepsilon_{x x}^{s i m}}+C_{\varepsilon, \varepsilon}\right)^{-1} .
$$

The numerical realisation of the previous algorithm can be performed in many distinct ways as summarised in $[19,20]$. The simplest one - the ensemble Kalman filter (EnKF) [6] -includes the representation of random variables via set of samples (socalled ensemble) such that:

$$
\mathbf{q}_{a}=\mathbf{q}_{f}+\mathbf{K}\left(\mathbf{e}_{x x}^{m e s}-\mathbf{e}_{x x}^{s i m}\right)
$$

holds. Here, $\mathbf{q}_{a}=\left[\ldots, q_{a}\left(\omega_{i}\right), \ldots\right]^{T}$ denotes the ensemble of posterior samples, whereas $\mathbf{q}_{f}, \mathbf{e}_{x x}^{\text {mes }}$ and $\mathbf{e}_{x x}^{s i m}$ are the ensembles of prior, the measurement and forecasted measurement, respectively. Note that the Kalman gain $\mathbf{K}$ is estimated directly from 
the ensemble, and hence represents only approximation of the Kalman gain given in Eq. (18).

The filter as presented in Eq. (17) is optimal when linearity and Gaussianity are assumed. In the nonlinear and non-Gaussian case, as considered in this paper, the filter delivers only sub-optimal solutions because only a piece of the available information is actually used in the assimilation process. Therefore, to allow for Gaussianity, all quantities of consideration are mapped from the non-Gaussian model to the Gaussian assimilation space by means of Gaussian anamorphosis [24, 23]. The anamorphosis denotes the nonlinear bijective transformations of the random variables $q$ and $\varepsilon_{x x}$

$$
\hat{q}=\mathscr{T}_{q}(q), \quad \hat{\varepsilon}=\mathscr{T}_{\varepsilon}\left(\varepsilon_{x x}\right)
$$

to the standard Gaussians $\hat{q}$ and $\hat{\varepsilon}$, respectively. The transformation implies

$$
\hat{Y}=\mathscr{T}_{\mathcal{\varepsilon}} \circ Y \circ \mathscr{T}_{q}^{-1},
$$

and results in a new EnKF estimate

$$
\hat{\mathbf{q}}_{a}=\mathscr{T}_{q}\left(\mathbf{q}_{f}\right)+\hat{\mathbf{K}}\left(\mathscr{T}_{\varepsilon}\left(\mathbf{e}_{x x}^{m e s}\right)-\mathscr{T}_{\varepsilon}\left(\mathbf{e}_{x x}^{s i m}\right)\right)
$$

which may be interpreted as some version of a pseudo-linearisation technique. Note that the quantity $\hat{\mathbf{q}}_{a}$ lives in assimilation space, and hence has to be transformed back to the model space by means of inverse transformation:

$$
\mathbf{q}_{a}=\mathscr{T}_{q}^{-1}\left(\hat{\mathbf{q}}_{a}\right) .
$$

The transformation can be performed in an analytical or numerical way depending on the type of the distributions $q$ and $\varepsilon_{x x}$ follow. For more information the reader is referred to [23].

\section{Numerical result comparison}

As reference, the numerical solution obtained with the finite element code Freefem++ [7] and the reference parameters:

$$
\left\{\begin{array}{l}
F_{e x}=10^{4} N, l_{F}^{e x}=3.75 m, c_{F}^{e x}=10 m / s, t_{F}^{e x}=0,1875 s \\
S_{b_{1}}^{e x}=S_{b_{2}}^{e x}=S_{b_{4}}^{e x}=S_{b_{5}}^{e x}=0,04 \& S_{b_{3}}^{e x}=0,03 \\
E_{c_{1}}^{e x}=E_{c_{2}}^{e x}=E_{c_{4}}^{e x}=E_{c_{5}}^{e x}=40 \mathrm{GPa} \& E_{c_{3}}^{e x}=30 \mathrm{GPa}
\end{array}\right.
$$



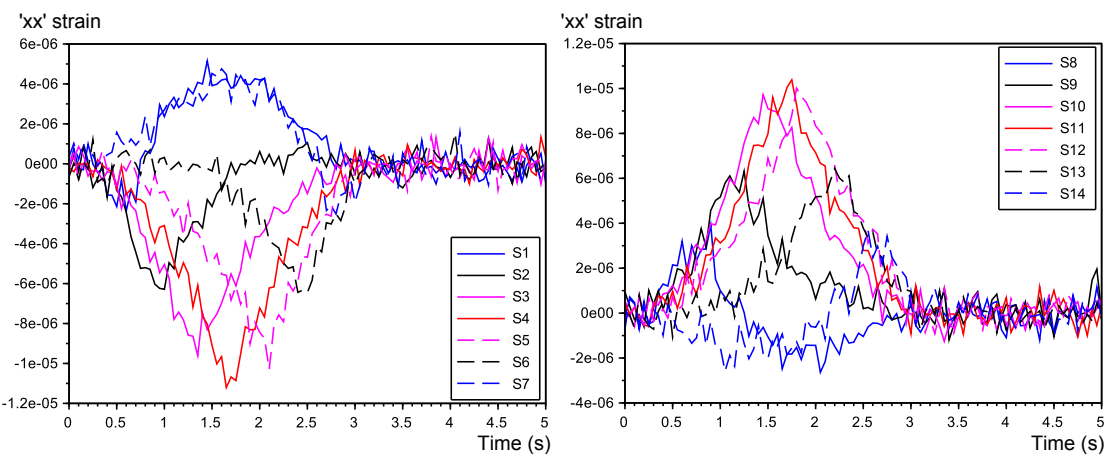

Figure 2: Corrupted sensor outputs $\varepsilon_{x x}^{\text {mes }}$ with Gaussian noise $\mathscr{N}\left(0, \sigma=5.10^{-7}\right)$

is considered. Note that the concrete subdomain 3 and the steel bar interval 3 are damaged. Herein, the corruputed sensor outputs $\varepsilon_{x x}^{m e s}$ are obtained from the perturbation of the reference numerical strain with a white noise $\mathscr{N}(0, \sigma)$. The time evolution of the corrupted sensor outputs for $\sigma=5 \cdot 10^{-7}$ can be seen in Fig. 2.

\subsection{Study 1: Identification of the moving load}

\subsubsection{Load identification using simple mechanical framework}

To get a better understanding of the load identification problem, the simple one dimensional and quasi-static mechanical model (see Fig. 3) is considered. In such a case one may easily get an analytical expression of the strain in quasi-static condi-

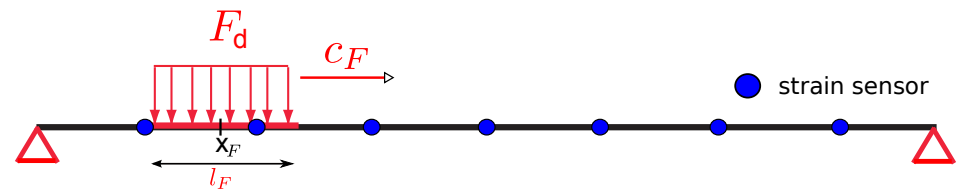

Figure 3: Simple mechanical model of the beam in quasi-static conditions 
tions:

$$
\varepsilon_{x x}(x, y)=\left\{\begin{array}{l}
l_{F} F_{d} E I\left(1-\frac{x_{F}}{L}\right) x y, x \in\left[0, x_{F}-l_{F} / 2[\right. \\
\frac{F_{d}}{2 E I}\left[2 l_{F}\left(1-\frac{x_{F}}{L}\right) x y-\left(x-x_{F}+\frac{l_{F}}{2}\right)^{2} y\right], x \in\left[x_{F}-l_{F} / 2, x_{F}+l_{F} / 2[\right. \\
\frac{l_{F} F_{d}}{E I} \frac{x_{F}}{L}(L-x) y, x \in\left[, x_{F}+l_{F} / 2, L\right]
\end{array}\right.
$$

where $x_{F}=c_{F} t$. Note that at the initial time the abscissa $x_{F}$ associated with the loading is located at the left beam support.

Let us remark that except in the loading zone $\left[x_{F}-l_{F} / 2, x_{F}+l_{F} / 2\right.$ [, the strain only depends on the total force obtained as product $l_{F} F_{d}$. In Fig. 4 are plotted isovalues of the data misfit functional for seven equidistributed sensors using the analytical expression in Eq. (25). From the graph above one may observe the lowest values of the

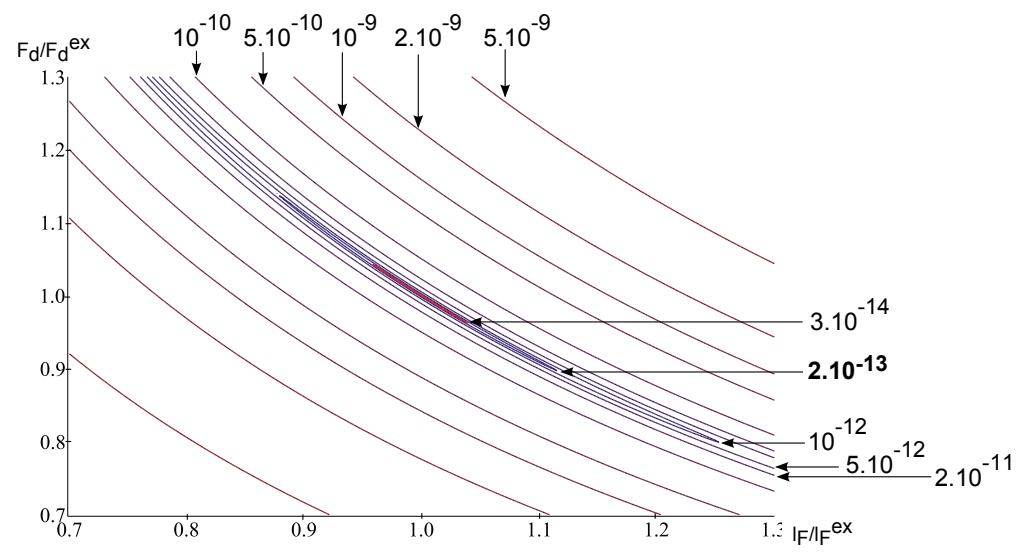

Figure 4: Data misfit functional $J\left(F_{d}, l_{F}\right)=\frac{1}{2} \sum_{i=1}^{7} \int_{0}^{T}\left[\varepsilon_{x x}\left(F_{d}, l_{F} ; x_{i}, t\right)-\left(\varepsilon_{x x}^{\text {mes }}\right)_{i}(t)\right]^{2} d t$ without regularization term $\left(\varepsilon_{1}^{\text {reg }}=0\right)$

functional in the close vicinity of the curve $F_{d} l_{F}=F_{d}^{e x} l_{F}^{e x}$. Thereby, one may confirm that the data misfit functional strongly depends on the total force. This leads to the conclusion that the total force can be properly identified, whereas the lineic force $F_{d}$ and the length force $l_{F}$ not. In fact, when the data misfit functional reaches the value $2.10^{-13}$ (see Fig. 4), the identification errors of $F_{d}$ and $l_{F}$ reach $10 \%$, whereas the identification error of the total force $F_{d} l_{F}$ amounts $1 \%$. 


\subsubsection{Load identification in 2D elastodynamics}

The initial values for the load identification are adopted to correspond to the dynamic loading due to the moving of a standard truck on the bridge (and thus on the beam). These values are used directly in the deterministic procedure, whereas the probabilistic approach uses the starting values in a form of a lognormal distribution with the mean equal to the starting values of the deterministic approach and the variance equal to $10 \%$ of the mean. These information is then assimilated with the measurement data obtained from sensors $S_{1}-S_{7}$ via the Tikhonov or ensemble Kalman filter procedure with 100 samples. The EnKF assimilation is performed in the transformed Gaussian space as described in the previous section. The anamorphosis function being employed is of the empirical kind and can be seen in Fig. 5 on the example of lineic force.

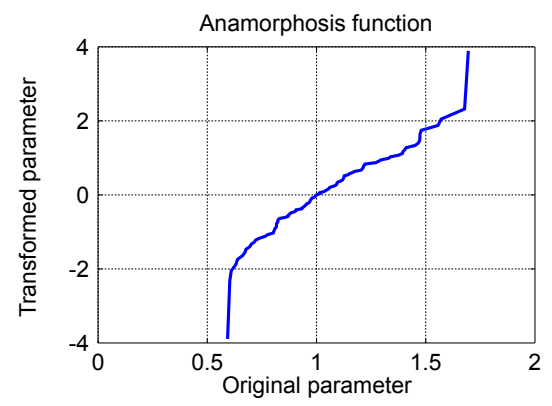

a) Parameter anamorphosis

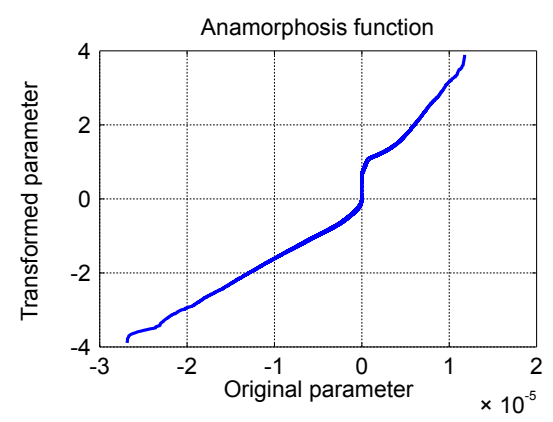

b) Measurement anamorphosis

Figure 5: Anamorphosis function for the lineic force

After assimilation of data, and mapping of the posterior to the model space, the EnKF procedure results in a posterior estimate similar to the one obtained by deterministic approach. For comparison purposes the mode of posterior is chosen to illustrate the EnKF result. In both probabilistic and deterministic procedures the lineic force and the length force are not properly estimated as already predicted in Section 4.1.1 on the simple 1D quasi-static mechanical model. Namely, the first one is underestimated $18 \%$ by EnKF (see Fig. 6) and 15\% by Tikhonov regularisation procedure (see Fig. 7) . Comparably, the length force is overstimated $20 \%$ by probabilistic and $15 \%$ by deterministic algorithm. In contrast to this, as depicted in Fig. 8, the total force obtained as a product of the lineic and length force is properly calibrated even when 


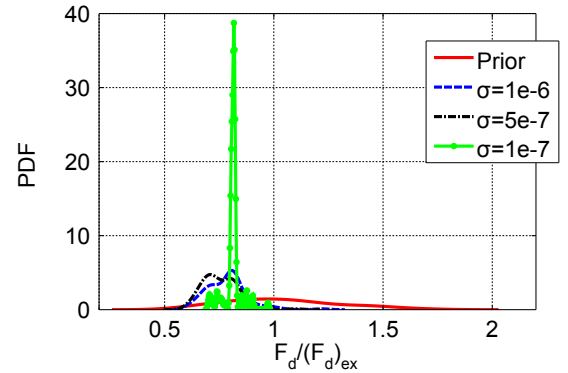

a) load amplitude

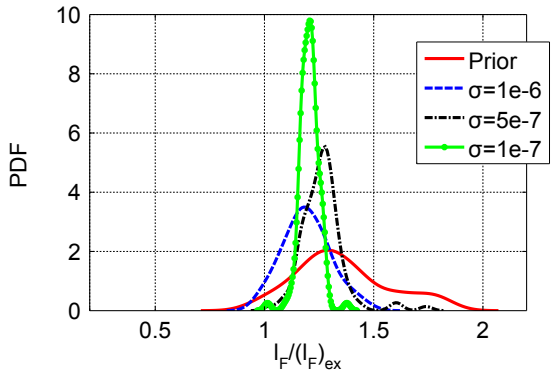

b) length force

Figure 6: Probabilistic identification of the load amplitude $F_{d}$ and length force $l_{F}$

the measurement error is high, i.e. $\sigma=10^{-6}$. Similarly, the velocity is identified within $2 \%$ of the error in both approaches.

Note that the probabilistic and deterministic estimates do not have the same meaning. The probabilistic is not only a point estimate, but a probability distribution as can be seen in Fig. 6 and Fig. 8. Thus, one also has to speak about the reduction of uncertainty going from prior to the posterior distribution. The reduction can be seen as a sort of measure of information gain. The stronger reduction the more information one gains from the data. This can be observed in Fig. 8b). However, note that the variance is underestimated due to nonlinearity of measurements. On the other side, when the reduction is not so strong such as for example in case of the steel bar cross section as described in Study 2, the posterior distribution is close to prior, and hence the information gain is small. This means that the measurement is not sensitive on the cross section or nonlinearity is too strong such that available information is not completely used in the process of updating. It is therefore likely that the minimisation error remains larger.

\subsection{Study 2: Determination of the material parameters}

In this study, the dynamic loading is considered known. As explained in Section 2.2, the concrete Young modulus $E_{c}$ (resp. the steel bar cross section $S_{b}$ ) is assumed constant on each of five subdomains (resp. on each of five intervals) (see Fig. 1). 


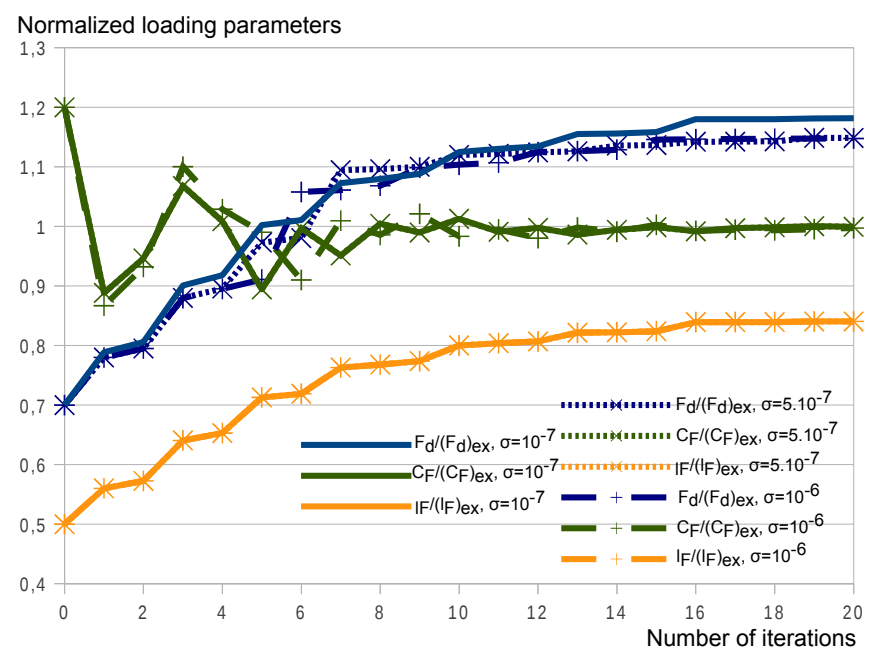

Figure 7: Load identification using the iterative deterministic approach

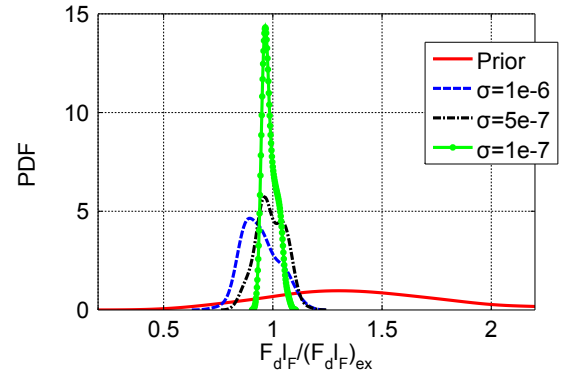

a) total force

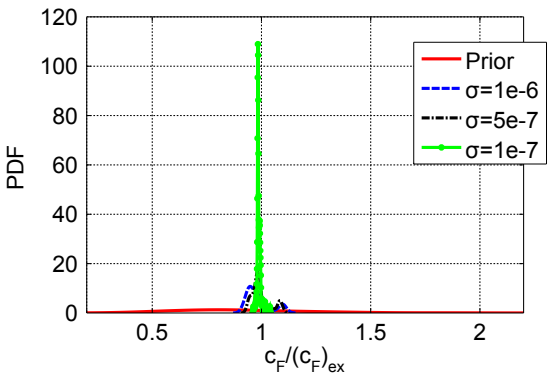

b) velocity

Figure 8: Probabilistic identification of total force $F_{d} l_{F}$ and velocity $c_{F}$ 
Similarly to the previous study, the starting values for the deterministic approach are taken to be the values characterising undamaged material. In the probabilistic framework, the identification of material parameters is done by assuming all of unknown quantities to be positive definite lognormal distributions with the mean equal to the deterministic values, and variance of $4 \%$ of the mean. The estimation is performed with the help of the Tikhonov regularisation/EnKF with 100 samples and whole time series of seven measurements read by $S_{8}-S_{14}$ sensors. Before the EnKF identification procedure, the parameter and the measurement are transformed to the standard Gaussians with the help of the anamorphosis function, see Fig. 9. The transformation is done locally for each unknown parameter and for each measurement point.
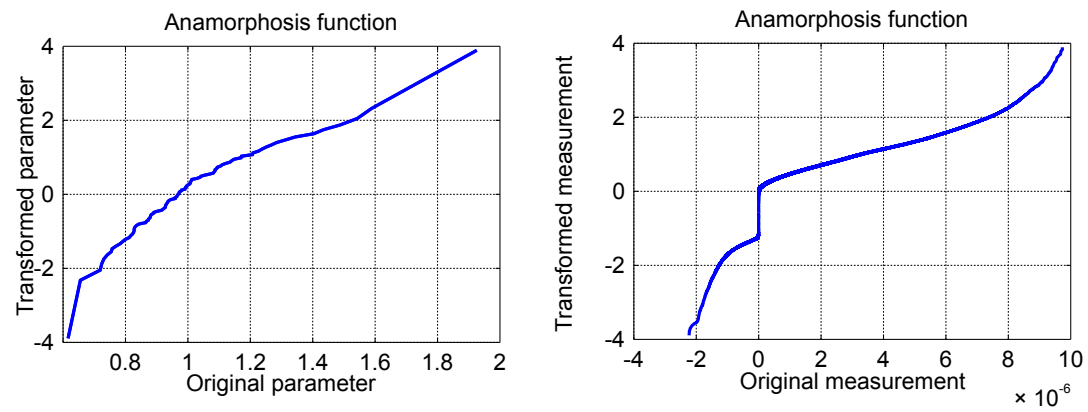

Figure 9: Identification of material parameters in section 3 by probabilistic approach

After assimilation, the posterior distributions of the material parameters are obtained. To compare these results with the deterministic approach, the mode is selected as representative. According to results shown in Tab. (1), both of approaches are able to detect and localize the concrete damage in the subdomain 3. The Young's modulus is estimated with an error of $8 \%$ in the deterministic case and $3 \%$ in the probabilistic approach. The probabilistic estimates do not fluctuate strongly with the increase of the measurement error, and hence the probabilistic approach seems to be more stable. Concerning the steel bar, Tab. (2) shows that both of approaches do not succeed in properly identifying the reduction of the cross section in the interval 3 . In Fig. 10, the posterior distributions of Young modulus $E_{c 3}$ and steel bar cross section $S_{b 3}$ are compared for different values of the measurement noise $\sigma$. As depicted, the posterior variances of both of parameters are smaller than the prior ones-the prior uncertainty is reduced. The reduction is not so much influenced by a measurement noise. However, the uncertainty reduction of Young's modulus is stronger than the corresponding one for the steel bar cross section. The reason is more than obvious. 


\begin{tabular}{|c|c|c|c|c|c|c|}
\hline & \multicolumn{2}{|c|}{$\sigma=10^{-7}$} & \multicolumn{2}{c|}{$\sigma=5.10^{-7}$} & \multicolumn{2}{c|}{$\sigma=10^{-6}$} \\
\hline Parameter & $\mathrm{D}$ & $\mathrm{P}$ & $\mathrm{D}$ & $\mathrm{P}$ & $\mathrm{D}$ & $\mathrm{P}$ \\
\hline$E_{c 1} /\left(E_{c 1}\right)_{e x}$ & 0.96 & 1.02 & 1. & 1.08 & 0.99 & 1.07 \\
\hline$E_{c 2} /\left(E_{c 2}\right)_{e x}$ & 1. & 1.06 & 1. & 1.06 & 1. & 0.94 \\
\hline $\boldsymbol{E}_{c 3} /\left(\boldsymbol{E}_{c 3}\right)_{e x}$ & $\mathbf{0 . 9 2}$ & $\mathbf{0 . 9 7}$ & $\mathbf{0 . 8 5}$ & $\mathbf{0 . 9 7}$ & $\mathbf{1 . 1 6}$ & $\mathbf{0 . 9 8}$ \\
\hline$E_{c 4} /\left(E_{c 4}\right)_{e x}$ & 1. & 1. & 0.95 & 1.0 & 1. & 1.01 \\
\hline$E_{c 5} /\left(E_{c 5}\right)_{e x}$ & 0.97 & 1. & 1. & 1.09 & 0.99 & 1.11 \\
\hline
\end{tabular}

Table 1: Updating of the concrete Young modulus - comparison of deterministic (D) and probabilistic $(P)$ approaches

\begin{tabular}{|c|c|c|c|c|c|c|}
\hline Parameter & $\mathrm{D}$ & $\mathrm{P}$ & $\mathrm{D}$ & $\mathrm{P}$ & $\mathrm{D}$ & $\mathrm{P}$ \\
\hline$S_{b 1} /\left(S_{b 1}\right)_{e x}$ & 0.98 & 1.00 & 1. & 1.02 & 0.99 & 1.01 \\
\hline$S_{b 2} /\left(S_{b 2}\right)_{e x}$ & 1. & 0.88 & 1. & 1.03 & 1. & 1.02 \\
\hline $\boldsymbol{S}_{b 3} /\left(\boldsymbol{S}_{b 3}\right)_{e x}$ & $\mathbf{1 . 2 5}$ & $\mathbf{0 . 9 7}$ & $\mathbf{1 . 3 3}$ & $\mathbf{0 . 9 5}$ & $\mathbf{1 . 2 7}$ & $\mathbf{1 . 1}$ \\
\hline$S_{b 4} /\left(S_{b 4}\right)_{e x}$ & 1. & 1.05 & 0.99 & 1. & 1. & 1.02 \\
\hline$S_{b 5} /\left(S_{b 5}\right)_{e x}$ & 0.99 & 1.08 & 1. & 1.01 & 0.99 & 1.01 \\
\hline
\end{tabular}

Table 2: Updating of the steel bar cross sections - comparison of deterministic (D) and probabilistic $(P)$ approaches

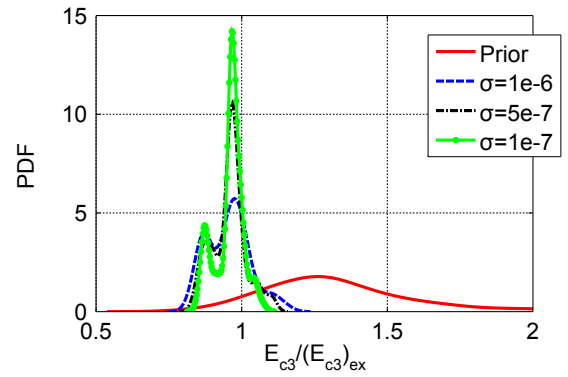

a) Young modulus

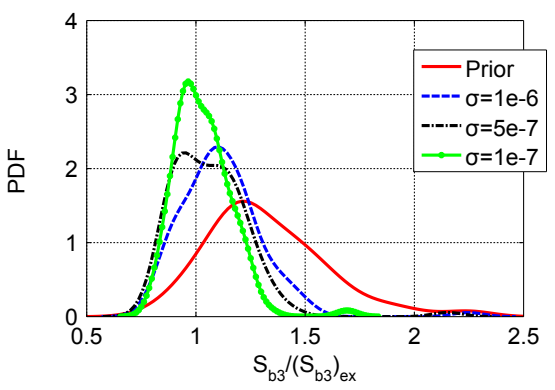

b) Steel cross section

Figure 10: Probabilistic identification of material parameters in section 3 


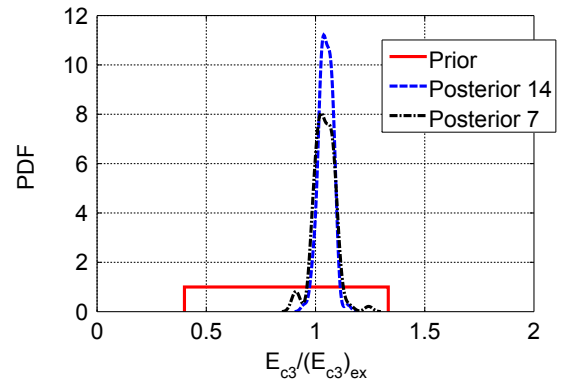

a) Young modulus

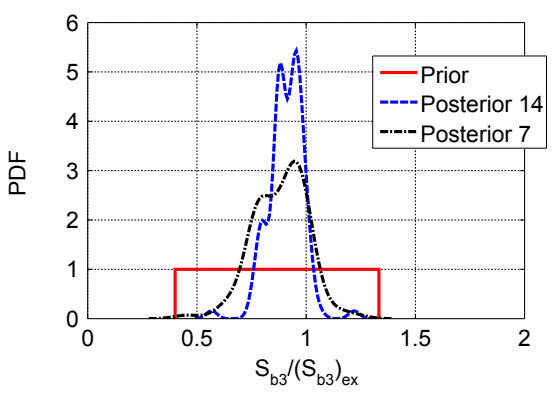

b) Steel bar cross section

Figure 11: Probabilistic identification of material parameters in section 3

Namely, the relation between the measurement and the cross section has higher degree of nonlinearity than the one for the Young's modulus. Hence, the difficulity in updating as previously observed by author in [20] on a heat diffusion example.

Finally, the prior assumption on the uncertainty of the cross section is not very much appropriate since the normalised version is bounded on both sides compared to the exact solution. Therefore, another, non-informative version of the prior is also investigated. By assuming the unknown parameters to follow the uniform distribution one obtains similar results as before, see Fig. 11. Only, this time the Young's modulus is slightly overestimated and the cross section is underestimated within $5 \%$ error. Note that assimilation with the help of all available measurements $S_{1}-S_{14}$ gives better estimates than the one when only sensors $S_{8}-S_{14}$ are included.

The estimation of material parameters is not optimal by EnKF because the assumptions of the linearity of the measurement operator and its Gaussianity fail. To be more close to these assumptions, the product of the Young's modulus and cross section (the axial rigidity of the bar) are assimilated with the measurement data. As expected, the assimilation results greatly improve. According to Fig. 12, the product is identified with very slight overestimation $(\leq 5 \%)$ in both cases. Note that this further can be improved by linearising the measurement as previously reported in [20] for the diffusion example.

Lastly, a comparison of the deterministic and the probabilistic approaches in terms of computation cost is performed. Having in mind that the process of solving the 


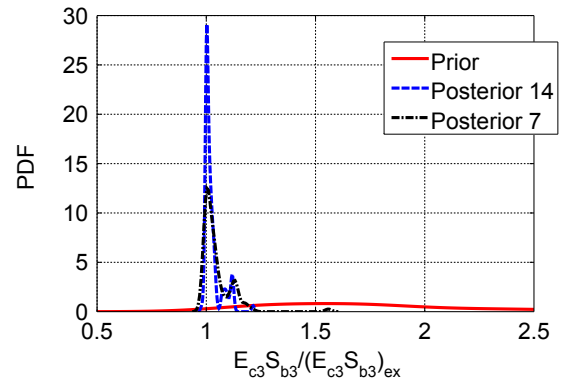

a) lognormal prior

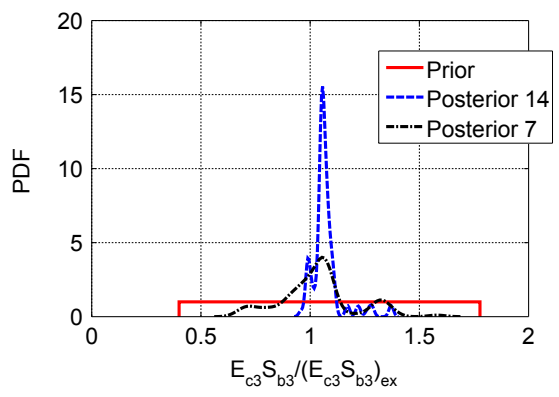

b) uniform prior

Figure 12: The estimation of the product of Young modulus and cross section

elastodynamics problem is the most expensive part of both identification algorithms, the cost is is given in terms of the number of elastodynamics solves. Let us note that in the deterministic approach, this total number includes direct and adjoint elastodynamics solves. Finaly both procedures require similar computation time. Indeed, to identify the loading parameters (resp. the material parameters), one needs about 100 solves.

\section{Conclusions}

The purpose of this paper was to study the qualitative differences between the probabilistic and deterministic approaches to determine load and material parameters considering corrupted sensor outputs. In the deterministic strategy, the inverse problem is solved by virtue of Tikhonov regularisation procedure, whereas in the probabilistic approach the Bayesian framework of regularisation is used. The results of this investigation show that for the same computation time both approaches can identify the loading velocity and the total force but not the individual loading characteristics, i.e. the loading amplitude and the surfacic force. The identification procedures are able to localise the concrete damage in the beam and to quantify it within an error of $15 \%$ even for highly noisy data. Nevertheless, the steel bar cross section area describing the corrosion effects remains difficult to identify. The reasons for this can be several: the measurement data are nonlinearly related to the unknown parameters and strain outputs may be less sensitive to the steel bar cross section. Even though both 
of approaches result in the same conclusion, there exist principal difference between them. The deterministic method is offering a single value estimate of the parameters, whereas the probabilistic method produces the probability estimate in a form of distribution. Hence, in the Bayesian framework a confidence interval on the updating parameters is obtained. Finally, note that both procedures rely on a regularisation parameter which is more or less subjectively chosen. To improve the existing estimates, the next step in the following research is to improve the model. Namely, the concrete is heterogeneous material which can be modelled as a random field or in a multiscale manner, and the loading is a stochastic process dependent on time. In addition to this, due to existing nonlinearities another kind of estimation procedure will be investigated such as for example the sequential or the nonlinear filter.

\section{Acknowledgements}

This work has been partially supported by the Region Ile de France and the German Research Foundation (DFG). Authors also thank the German-French School of Computational Engineering on Virtual Materials and their Validation (ViVaCE) for the cooperation.

\section{References}

[1] Ludovic Chamoin, Pierre Ladevèze, and Julien Waeytens. Goal-oriented updating of mechanical models using the adjoint framework. Computational Mechanics, page 17p, 2014.

[2] A. Deraemaeker, P. Ladevèze, and P. Leconte. Reduced bases for model updating in structural dynamics based on constitutive relation error. Computer Methods in Applied Mechanics and Engineering, 191:2427-2444, 2002.

[3] A. Deraemaeker, E. Reynders, G. De Rock, and J. Kullaa. Vibration-based structural health monitoring using output-only measurements under changing environment. Mechanical Systems and Signal Processing, 22:34-56, 2008. 
[4] S.W. Doebling, C.R. Farrar, and M.B. Prime. A summary review of vibrationbased damage identification methods. Identification Methods, The Shock and Vibration Digest, 30:91-105, 1998.

[5] G. A. Einicke. Smoothing, Filtering and Prediction: Estimating the Past, Present and Future. Rijeka, Croatia: Intech, 2012.

[6] G. Evensen. Data Assimilation, The Ensemble Kalman Filter. Springer, Berlin, June 2007.

[7] F. Hecht. New development in freefem++. J. Numer. Math., 20(3-4):251-265, 2012.

[8] B. Jacob, E.J. O'Brien, and W. Newton. Assessment of the accuracy and classification of Weigh-in-Motion systems: Part 2 European specification. International Journal of Vehicle Design, 7:153-168, 2000.

[9] A. Kučerová, J. Sýkora, B. Rosić, and H. G. Matthies. Acceleration of uncertainty updating in the description of transport processes in heterogeneous materials. Journal of Computational and Applied Mathematics, 236(18):48624872, 2012.

[10] P. Ladevèze and A. Chaouaki. Application of a posteriori error estimation for structural model updating. Inverse Problems, 15:49-58, 1999.

[11] A. Litvinenko and H. G. Matthies. Uncertainty quantification and non-linear bayesian update of pce coefficients. PAMM, 13(1):379-380, 2013.

[12] D. G. Luenberger. Optimization by Vector Space Methods. John Wiley and Sons, Inc., New York, 1969.

[13] Y. M. Marzouk and H. N. Najm. Dimensionality reduction and polynomial chaos acceleration of Bayesian inference in inverse problems. Journal of Computational Physics, 228(6):1862-1902, 2009.

[14] J. Mazars. A description of micro- and macroscale damage of concrete structures. Engineering Fracture Mechancis, 25:729-737, 1986.

[15] V. A. Morozov. On the solution of functional equations by the method of regularization. Soviet Math. Dokl., 7:414-417, 1966. 
[16] F. Moses. Weigh-In-Motion system using instrumented bridges. Transportation Engineering, 105:233-249, 1979.

[17] E.J. O'Brien, A. Znidaric, and A. Dempsey. Comparison of two independently developed bridge weigh-in-motion systems. International Journal of Vehicle Design, 61:147-162, 1999.

[18] D. Posenato, P. Kripakaran, D. Inaudi, and I.F.C Smith. Methodologies for model-free data interpretation of civil engineering structures. Computers and Structures, 88:467-482, 2010.

[19] B. Rosić, A. Kučerová, J. Sýkora, O. Pajonk, A. Litvinenko, and H. G. Matthies. Parameter identification in a probabilistic setting. Engineering Structures, 50:179-196, 2013.

[20] B. Rosić, J. Sýkora, O. Pajonk, A. Kučerová, and H. G. Matthies. Numerical approaches to bayesian updating of uncertainty. Technical report, Institut für Wissenschaftliches Rechnen, TU Braunschweig, 2014.

[21] A. Rytter. Vibrational based inspection of civil engineering structures. PhD thesis, University of Aalbork, Denmark, 1993.

[22] J.P. Santos, C. Crémona, A. Orcesi, and P. Silveira. Multivariate statistical analysis for early damage detection. Engineering Structures, 56:273-285, 2013.

[23] A. Schöniger, W. Nowak, and H.-J. Hendricks Franssen. Parameter estimation by ensemble kalman filters with transformed data: Approach and application to hydraulic tomography. Water Resources Research, 48(4):n/a-n/a, 2012.

[24] E. Simon and L. Bertino. Application of the gaussian anamorphosis to assimilation in a 3-d coupled physical-ecosystem model of the north atlantic with the enkf: a twin experiment. Ocean Science, 5(4):495-510, 2009.

[25] A. Tarantola. Inverse Problem Theory and Methods for Model Parameter Estimation. Society for Industrial and Applied Mathematics, 2005.

[26] A.N. Tikhonov and Y. Arsenin. Solutions to ill-posed problems. WintsonWidley, New York, 1977.

[27] Curtis R. Vogel. Computational Methods for Inverse Problems. Society for Industrial and Applied Mathematics, Philadelphia, PA, USA, 2002. 
[28] Julien Waeytens, Veronique Le Corvec, Philippe Lévèqua, Dominique Siegert, and Frédéric Bourquin. Elastodynamics model updating for the monitoring of reinforced concrete beam: methodology and numerical implementation. Applied Mechanics and Materials, 513-517:3401-3406, 2014. 
Technische Universität Braunschweig

Informatik-Berichte ab Nr. 2012-03

2012-02 B. V. Rosić and H. G. Matthies

2012-03 J. Rang

2012-04 S. Kolatzki, M. Hagner, U. Goltz and A. Rausch

2012-05 M. Espig, W. Hackbusch, A. Litvinenko, H. G. Matthies and P. Wähnert

2012-06 S. Mennike

2012-07 S. Lity, R. Lachmann, M. Lochau, I. Schaefer

2013-01 M. Lochau, S. Mennicke, J. Schroeter und T. Winkelmann

2013-02 S. Lity, M. Lochau, U. Goltz

2013-03 L. Giraldi, A. Litvinenko, D. Liu, H. G. Matthies, A. Nouy
Stochastic Plasticity - A Variational Inequality Formulation and Functional Approximation Approach I: The Linear Case

An analysis of the Prothero-Robinson example for constructing new DIRK and ROW methods

A Formal Definition for the Description of Distributed Concurrent Components - Extended Version

Efficient low-rank approximation of the stochastic Galerkin matrix in tensor formats

A Petri Net Semantics for the JoinCalculus

Delta-oriented Software Product Line Test Models - The Body Comfort System Case Study

Extended Version of 'Automated Verification of Feature Model Configuration Processes based on Workflow Petri Nets'

A Formal Operational Semantics of Sequential Function Tables for Model-based SPL Conformance Testing

To be or not to be intrusive? The solution of parametric and stochastic equations - the "plain vanilla" Galerkin case 
2013-04 A. Litvinenko, H. G. Matthies

2013-05 J. Rang

2013-06 J. Koslowski

2014-01 B. Rosić, J. Diekmann

2014-02 M. Krosche, W. Heinze

2014-03 J. Rang

2014-04 S. Mennicke,

J.-W. Schicke-Uffmann,

U. Goltz

2014-05 A. Martens, C. Borchert, T. O. Geissler, O. Spinzcyk, D. Lohmann, R. Kapitza

2014-06 J. Rang

2014-07 J. Rang, R. Niekamp

2014-08 J. Rang

2014-09 J. Wayetens, B. V. Rosić
Inverse problems and uncertainty quantification

Improved traditional RosenbrockWanner methods for stiff ODEs and DAEs

Deterministic single-state 2PDAs are Turing-complete

Stochastic Description of Aircraft Simulation Models and Numerical Approaches

A Robustness Analysis of a Preliminary Design of a CESTOL Aircraft

Apdative timestep control for fully implicit Runge-Kutta methods of higher order

Free-Choice Petri Nets and Step Branching Time

Exploiting determinism for efficient protection against arbitrary state corruptions

An analysis of the Prothero-Robinson example for constructing new adaptive ESDIRK methods of order 3 and 4

A component framework for the parallel solution of the incompressible Navier- Stokes equations with RadauIIA methods

The Prothero and Robinson example: Convergence studies for Runge-Kutta and Rosenbrock-Wanner methods

Comparison of deterministic and probabilistic approaches to identify the dynamic moving load and damages of a reinforced concrete beam 